\title{
REFLETINDO SOBRE A IMPORTÂNCIA DO CUIDADO EM ENFERMAGEM À CRIANÇA COM OBESIDADE NO BRASIL: UMA REVISÃO
}

\author{
REFLECTING ON THE IMPORTANCE OF NURSING CARE FOR \\ CHILDREN WITH OBESITY IN BRAZIL: A REVIEW
}

Joselane Izaquiel Marinho ${ }^{1}$

Larissa Soares Mariz ${ }^{2}$

\begin{abstract}
RESUMO: A obesidade é entendida como o excesso de gordura corporal, a qual é considerada um problema da sociedade moderna que atinge em elevadas proporções à população infantil. É um problema mundial e um problema de saúde pública uma vez que vem crescendo em números exponenciais e atinge todas as faixas etárias e classes sociais. Dessa forma, o presente estudo tem como objetivo refletir sobre a importância do cuidado de enfermagem à criança com obesidade, tendo em vista à prevenção/promoção e consequentemente a diminuição da incidência da obesidade infantil. Trata-se de um estudo de uma revisão integrativa da literatura, que analisa estudos desenvolvidos mediante diferentes metodologias, permitindo aos revisores compilar resultados, a fim de reunir o conhecimento préexistente sobre a temática. Como resultados foi possível perceber a importância da enfermagem frente ao cuidado à criança obesa bem como, de educador em saúde e participante da equipe multiprofissional de saúde. Por conseguinte, deve-se intervir e possibilitar uma melhor qualidade de vida a população, buscando apoiar a família, a escola e a comunidade para que todos possam participar ativamente da diminuição da obesidade infantil, prevenindo-os dos riscos e impedindo essas crianças de se tornarem um adulto obeso, e consequentemente vítima de outros agravos.
\end{abstract}

Palavras chave: Criança. Enfermagem. Obesidade.

ABSTRACT: Obesity is understood as excess body fat, which is considered a problem in modern society that reaches high proportions to the child population. It is a problem worldwide, since the significant increase in overweight and obesity affects all age groups, including children, and social classes, which is considered a major

\footnotetext{
${ }^{1}$ Graduanda do curso de bacharelado em Enfermagem da Universidade Federal de Campina Grande.

${ }^{2}$ Orientadora/Dra ${ }^{\mathrm{a}}$. do Curso Bacharelado em Enfermagem da Universidade Federal de Campina Grande, do Centro de Educação e Saúde. E-mail: marinhojoselane96@gmail.com.
} 
public health problem. Thus, the present study aims to inform the reader about the importance of nursing care for children with obesity, with a view to prevention / promotion and, consequently, to reducing the incidence of childhood obesity. In addition, this study consists of an integrative literature review, which brings together findings from studies developed using different methodologies, allowing reviewers to compile results, which aims to gather pre-existing knowledge on the subject. Obtaining as a result the conception of the importance of the role of nursing freight to care for obese children as well as, of health educator and participant of the multidisciplinary health team. Therefore, the population must intervene and provide a better quality of life, seeking to support the family, the school and the community so that everyone can actively participate in the reduction of childhood obesity, preventing them from risks and preventing these children from becoming one. Obese adult, and consequently a victim of other diseases.

Keywords: Kids. Nursing. Obesity. 


\section{INTRODUÇÃO}

O Brasil vem sofrendo com um aumento expressivo de sobrepeso e obesidade em todas as faixas etárias e classes sociais, o que é considerado um grande problema de saúde pública (CARVALHO et. al., 2013). Segundo a Organização Mundial da Saúde (OMS), a obesidade é constituída pelo aumento de gordura corporal, provocado pelo balanço energético positivo. Esse fenômeno contribui em risco para a saúde do indivíduo devido às complicações metabólicas. Dentre essas complicações, está o aumento da pressão arterial, diabetes mellitus, aumento nos níveis de colesterol e triglicerídeos (BRASIL, 2014).

Com isso, entende-se por obesidade como o excesso de gordura corporal, a qual é considerada um problema da sociedade moderna que atinge elevadas proporções à população infantil (SILVA et. al., 2008).

Desse modo, nota-se, que a obesidade está afetando inclusive as crianças, como mostra a Pesquisa de Orçamento Familiar (2009) que analisou o estado nutricional e antropométrico da população brasileira e observou alterações no perfil da população. Nesta pesquisa, evidenciou-se que o excesso de peso e obesidade tiveram altos índices, apresentando $51,4 \%$ nos meninos e $43,8 \%$ nas meninas na faixa etária entre 5 a 9 anos de idade. Ressalta-se que nessas crianças com excesso de peso citadas anteriormente, nas meninas, $32 \%$, estavam com sobrepeso e $11,8 \%$, obesas. Já nos meninos, o percentual foi maior com $34,8 \%$ de sobrepeso e 16,6\% de obesidade (IBGE, 2011).

À vista disso, a obesidade está relacionada a numerosos fatores que desequilibram o balanço energético. Entre estes estão os genéticos, onde pais e mães que são obesos seus filhos herdam esta característica. Contudo, a maioria das variáveis causadoras da obesidade é por ingestão demasiada de alimentos calóricos (SANTOS, 2013), inferindo diretamente na qualidade de vida da população.

Embora, os fatores genéticos predisponham o desenvolvimento da obesidade, os principais determinantes de seu aumento parecem ser os fatores ambientais e 
comportamentais, como a falta de atividade física (REY-LÓPEZ et al., 2008) e o maior tempo assistindo à televisão (FULTON et al., 2009). A Organização Mundial da Saúde (OMS) estima que o excesso de peso atinja aproximadamente 42 milhões de crianças menores de cinco anos de idade, sendo a maioria residente de países em desenvolvimento.

Tendo em vista o impasse mundial da obesidade o desenvolvimento infantil, necessita ser acompanhado e assistido pelo profissional de enfermagem, para detecção precoce, diagnóstico e acompanhamento, do sobre peso existente nas crianças para que se inicie o tratamento de forma adequada buscando resultados satisfatórios e eficazes até a fase adulta. Tornando-se imprescindível a avaliação rotineira das crianças, no que se refere ao acompanhamento de peso e altura, bem como, levando em consideração os diferencias de desenvolvimento infantil por gênero, visando o bem-estar e a saúde da criança, a prática de acompanhamento diária nas Unidades de Saúde como estratégia para se combater a obesidade infantil (MARCHI-ALVES et. al., 2011).

Assim, no cenário brasileiro de saúde, os enfermeiros têm como atribuições estabelecer a estratificação do risco para obesidade, realizar ações de promoção à saúde com participação social, apoiar o autocuidado, bem como, promover a prestação de cuidado aos indivíduos que já se encontram com obesidade, principalmente se houver morbidades associadas. Além disso, devem acompanhar aqueles que se submeteram a procedimentos cirúrgicos relacionados à obesidade (BRASIL, 2012). Ademais, o enfermeiro como membro da equipe multidisciplinar deve reconhecer a sua importância na atuação de redução da incidência através da promoção e prevenção e, portanto, diminuição dos danos e/ou alterações no estilo de vida das crianças devido ao excesso de peso.

Para MARCHI-ALVES et. al., (2011) e ARAÚJO et. al., (2012) o acompanhamento das crianças torna-se indispensável no atendimento de enfermagem, com isso pode-se detectar precocemente a obesidade infantil e tratá-lo com mais eficiência. Essa detecção ocorre a partir do peso e altura, sendo assim, um dos critérios primordiais para avaliação do estado de saúde da criança.

Diante disso, o presente estudo tem como objetivo refletir a base dos estudos existentes sobre a importância do cuidado de enfermagem à criança com obesidade 
no Brasil, tendo em vista a prevenção/promoção e consequentemente a diminuição da incidência da obesidade infantil no país.

\section{MATERIAIS E MÉTODO}

Consiste em um estudo do tipo revisão integrativa da literatura que teve por finalidade reunir o conhecimento pré-existente sobre a temática do estudo.

A revisão integrativa configura-se, portanto, como um tipo de revisão da literatura que aglomera achados de estudos desenvolvidos mediante diferentes metodologias, permitindo aos revisores compilar resultados (SOARES et. al., 2014).

O estudo foi desenvolvido em Fevereiro de 2019, no cenário virtual da Biblioteca Virtual de Saúde (BVS) que possui indexadas as seguintes bases de dados: Medical Literature Analysisand Retrieval System Online (MEDLINE), Literatura Latino Americana e do Caribe em Ciências da Saúde (LILACS), na biblioteca eletrônica Scientific Electronic Library Online (SciELO) e na Base de Dados de Enfermagem (BDENF).

Foram utilizados os descritores: obesidade, criança, enfermagem, os descritores foram devidamente consultados nos Descritores em Ciências da Saúde (DeCS). Após o levantamento das publicações, 45 artigos inicialmente foram selecionados e os resumos, lidos e analisados. Depois dessa análise inicial, foram selecionados os estudos que abordaram o cuidado em enfermagem à criança com obesidade. Os critérios de inclusão foram artigos disponíveis na íntegra no período de 05 anos (2014-2019); nos idiomas português, inglês e espanhol, publicados nas referidas bases de dados. $\mathrm{E}$ os critérios de exclusão foram artigos repetidos nas bases de dados, que foram classificados como editoriais ou cartas ao editor, e que abordavam a obesidade infantil fora dos cuidados de enfermagem.

Após analisados os resumos, os artigos foram lidos na íntegra, e dos artigos encontrados foram selecionados segundo critérios de inclusão e exclusão 10 (dez) pesquisas. Posteriormente, foi construído um instrumento para integração dos achados em forma de quadro de modo a dar visibilidade às principais características 
de cada produção (título, autor/ano de publicação, objetivo e principais resultados), mantendo-se a autenticidade das ideias, conceito e definições dos autores.

\section{RESULTADOS}

Após a fase de seleção dos artigos, foram incluídos na revisão integrativa 10 (dez) estudos. Estes foram organizados e dispostos no quadro 01 a seguir:

Quadro 01: Descrição dos artigos selecionados conforme título, autor/ano de publicação, objetivos e principais resultados.

\begin{tabular}{|c|c|c|c|}
\hline TÍTULO/ & $\begin{array}{c}\text { AUTOR/ANO } \\
\text { DE } \\
\text { PUBLICAÇÃO }\end{array}$ & OBJETIVO & PRINCIPAIS RESULTADOS \\
\hline $\begin{array}{ll}\text { Ações } & \text { de } \\
\text { enfermeiros } & \epsilon \\
\text { professores } & \text { na } \\
\text { prevenção e nc } \\
\text { combate } \\
\text { obesidade } \\
\text { infantil. }\end{array}$ & & $\mid \begin{array}{l}\text { Conhecer como os } \\
\text { enfermeiros } \\
\text { professores } \\
\text { contribuem } \\
\text { prevenção } \\
\text { combate } \\
\text { obesidade infantil. }\end{array}$ & $\begin{array}{l}\text { Os enfermeiros descreveram } \\
\text { como fatores que contribuem } \\
\text { para a obesidade na criança a } \\
\text { ingestão de alimentos pouco } \\
\text { saudáveis e a cultura das } \\
\text { famílias. Apontaram que não } \\
\text { adianta intervir sobre a } \\
\text { criança uma vez que os } \\
\text { professores atribuem à } \\
\text { obesidade infantil à ingestão } \\
\text { de alimentos inapropriados } \\
\text { principalmente pelo que } \\
\text { observaram nos intervalos de } \\
\text { aula. }\end{array}$ \\
\hline $\begin{array}{l}\text { Ações } \\
\text { Enfermagem } \\
\text { combate } \\
\text { obesidade } \\
\text { infantil } \\
\text { período escolar. }\end{array}$ & $\begin{array}{l}\text { la } \\
\text { ànIBEIRO K.R./ } \\
\text { et al., } 2015 .\end{array}$ & $\begin{array}{l}\text { Discutir por meio } \\
\text { da literatura sobre } \\
\text { a obesidade infantill } \\
\text { bem como seus } \\
\text { métodos } \\
\text { preventivos e as } \\
\text { ações } \\
\text { desenvolvidas pela } \\
\text { equipe } \\
\text { enfermagem frente } \\
\text { ao problema no } \\
\text { ambiente escolar. }\end{array}$ & $\begin{array}{l}\text { No Ambiente } \text { Escolar a } \\
\text { obesidade reflete diretamente } \\
\text { nas crianças afetadas, } \\
\text { atingindo sua autoestima } \\
\text { limitando sua capacidade de } \\
\text { realizar atividades físicas } \\
\text { promovidas pelos professores. } \\
\text { É fundamental que o } \\
\text { enfermeiro como educador, } \\
\text { participe diretamente da } \\
\text { prevenção e tratamento da } \\
\text { mesma no ambiente escolar } \\
\text { criando ranecimentos }\end{array}$ \\
\hline
\end{tabular}




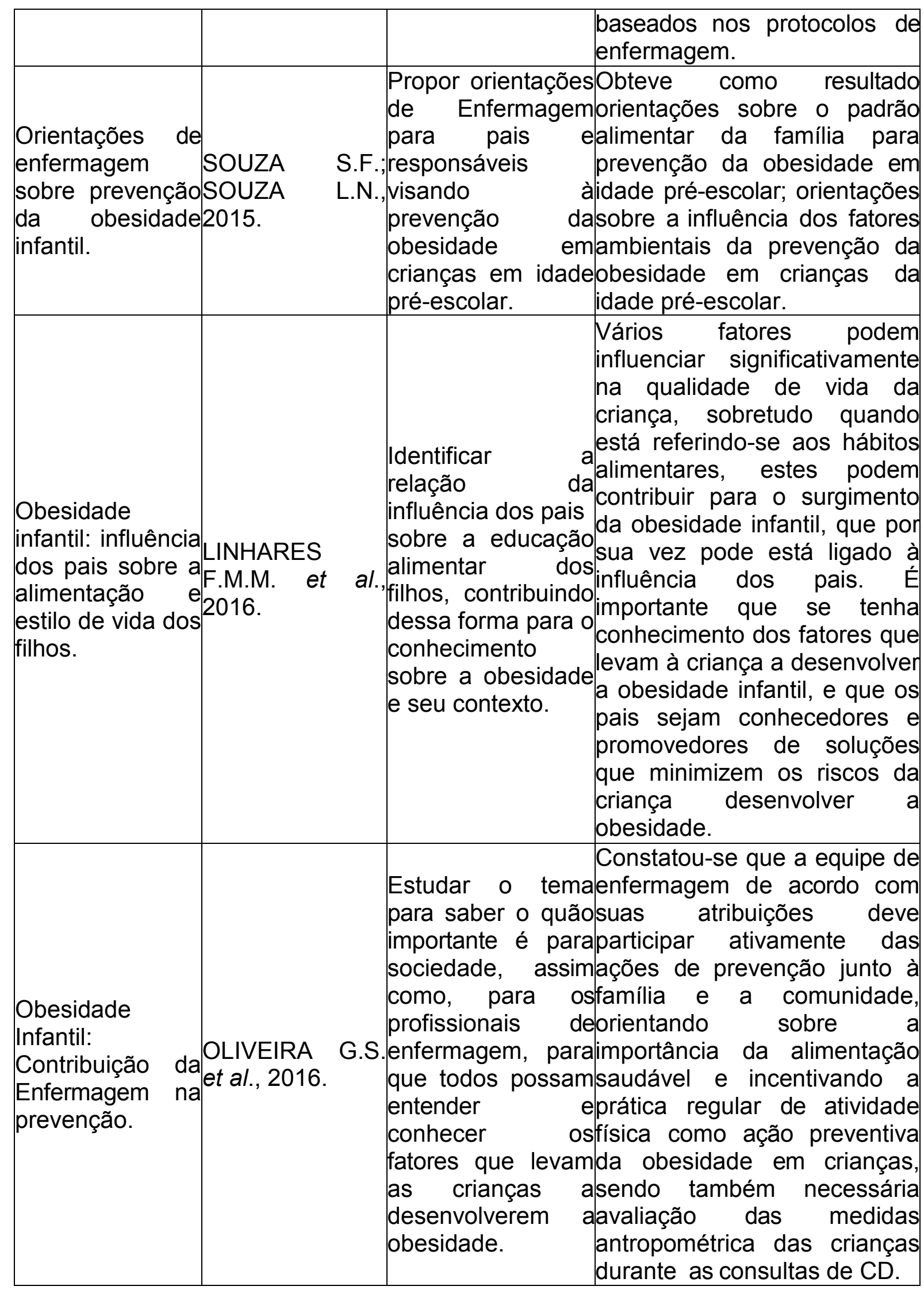




\begin{tabular}{|c|c|}
\hline $\begin{array}{lr}\text { Assistência } & \text { de } \\
\text { enfermagem } & \text { na } \\
\text { prevenção } & \text { e } \\
\text { promoção } & \text { dalPINHO G.B.P. } \\
\text { Saúde } & \text { dalGODINHO } \\
\text { criança } & \text { eJ.S.L., } 2017 . \\
\text { adolescente } & \text { com } \\
\text { risco } & \text { para } \\
\text { obesidade. } & \end{array}$ & 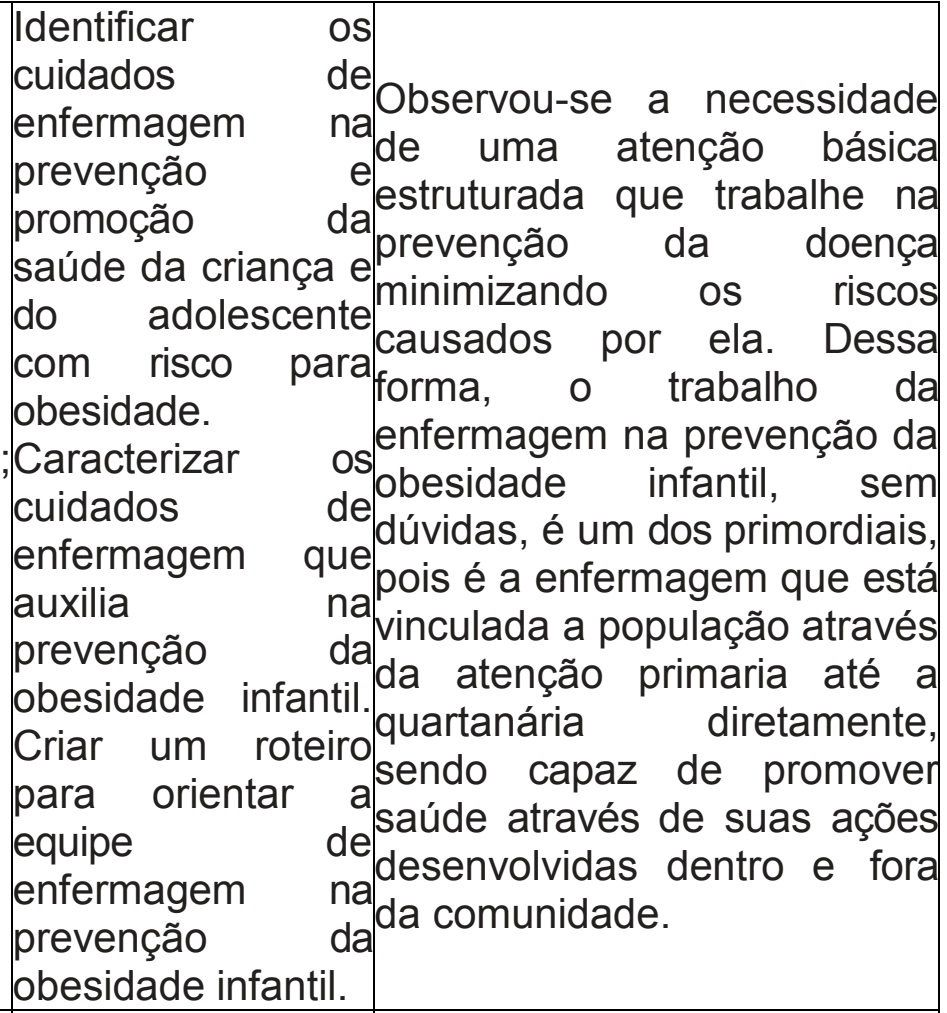 \\
\hline $\begin{array}{lr}\text { Intervenções } & \text { do } \\
\text { enfermeiro } & \text { às } \\
\text { pessoas r com } & \text { coma } \\
\text { obesidade r na } \\
\text { Atenção Primária } \\
\text { à Saúde: revisão } \\
\text { integrativa. }\end{array}$ & 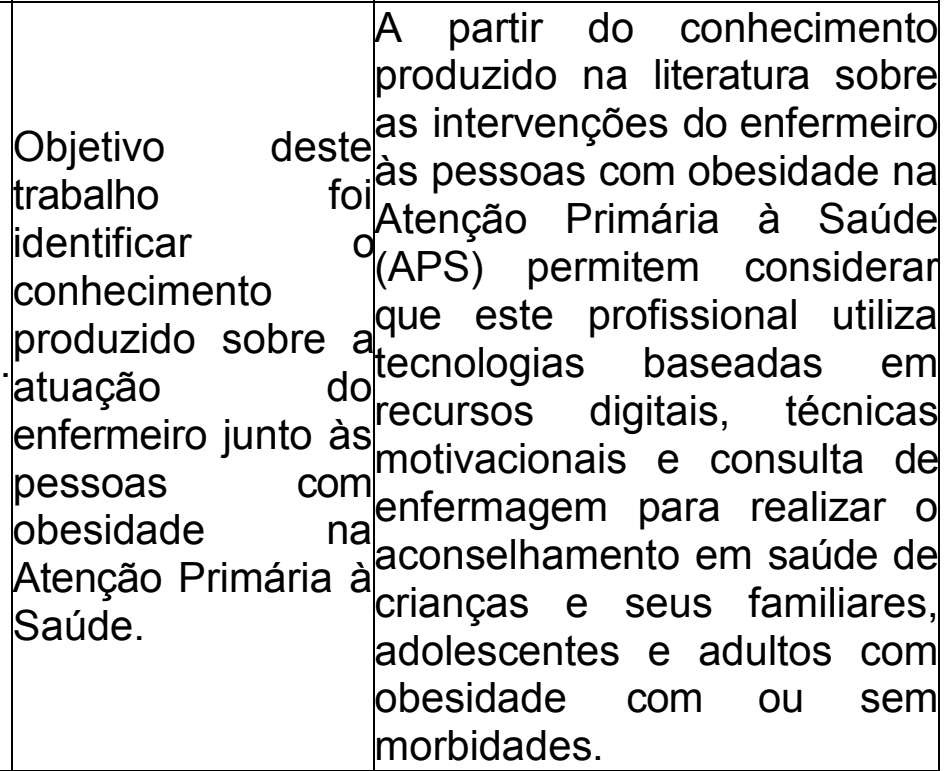 \\
\hline $\begin{array}{l}\text { Fatores de riscos } \\
\text { metabólicos } \\
\text { crianças } \\
\text { atenção primária } \\
\text { à saúde. }\end{array}$ & 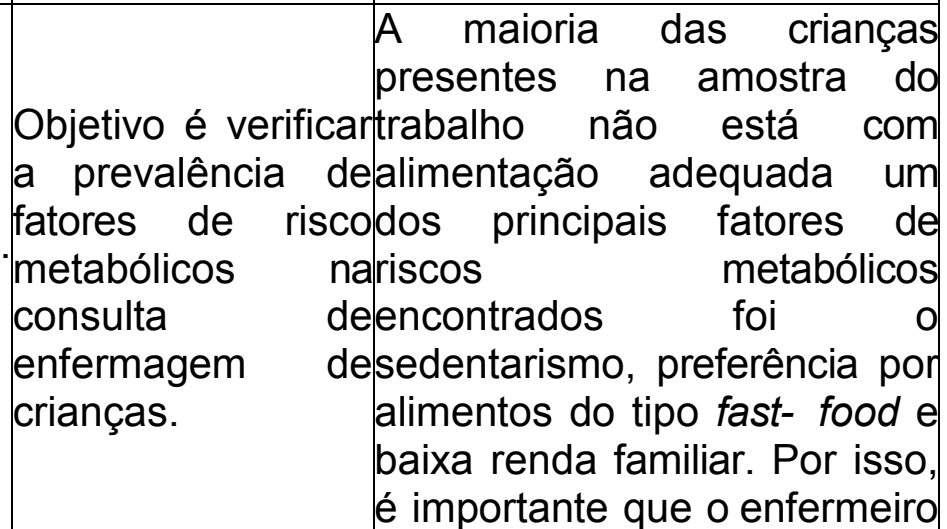 \\
\hline
\end{tabular}




\begin{tabular}{|c|c|c|}
\hline & & $\begin{array}{l}\text { contribua na educação em } \\
\text { saúde, com a promoção de } \\
\text { ambientes } \\
\text { investigando a saudáveis, } \\
\text { criança e do responsável } \\
\text { durante as consultas de } \\
\text { enfermagem. }\end{array}$ \\
\hline $\begin{array}{ll}\text { Assistência } & \text { de } \\
\text { Enfermagem } & \text { na } \\
\text { Obesidade } & \\
\text { Infantil: } & \text { Uma } \\
\text { Revisão } & \\
\text { Integrativa. } & \end{array}$ & $\begin{array}{l}\text { a } \\
\text { aLVES N.S } \\
\text { FAUSTINO } \\
\text { F.K.A., 2019. }\end{array}$ & $\begin{array}{l}\text { Este estudo tem } \\
\text { como objetivoÉ necessário criar estratégias } \\
\text { verificar aefetivas para a redução da } \\
\text { assistência deobesidade infantil de forma a } \\
\text { enfermagem naevitar futuros adultos obesos } \\
\text { obesidade infantile doentes. É de suma } \\
\text { além de investigarimportância que o enfermeiro } \\
\text { as principaisseja capacitado para atuar na } \\
\text { complicações e opromoção e prevenção de } \\
\text { impacto dasaúde, para ofertar uma } \\
\text { obesidade infantilassistência de enfermagem } \\
\text { na saúde daadequada e de qualidade. } \\
\text { criança. }\end{array}$ \\
\hline $\begin{array}{ll}\text { Saúde } & \text { do } \\
\text { escolar: } & \\
\text { Enfermagem } & \text { na } \\
\text { prevenção } & \text { da } \\
\text { obesidade } & \\
\text { infantil } & \text { no } \\
\text { contexto escolar }\end{array}$ & $\begin{array}{l}\text { SILVA } \\
2019 .\end{array}$ & 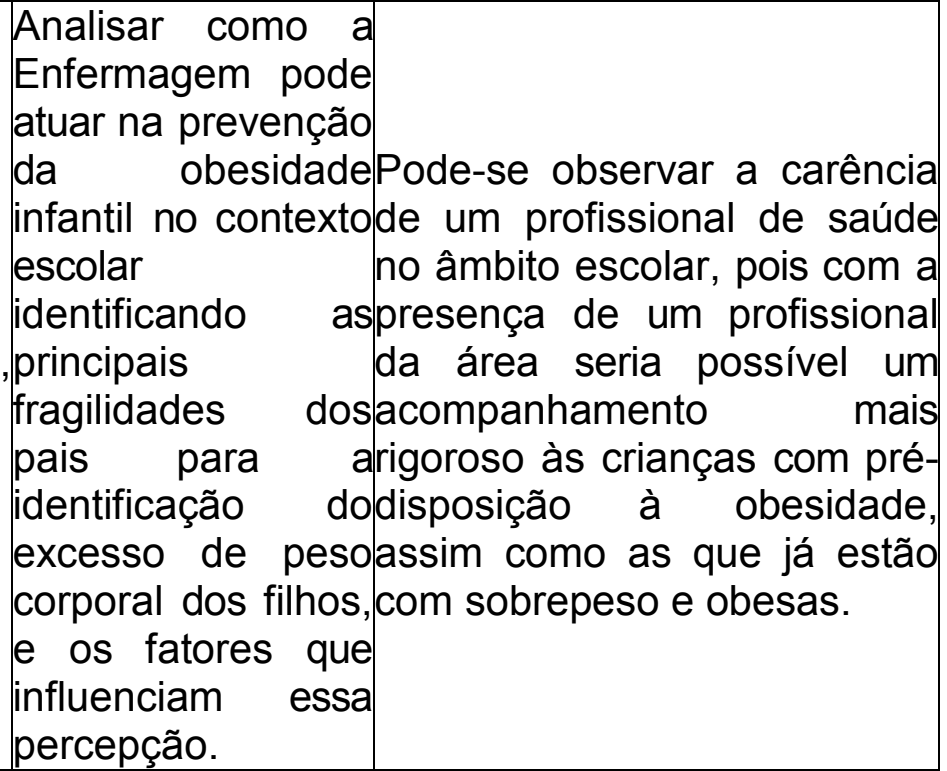 \\
\hline
\end{tabular}

\section{DISCUSSÃO}

É de extrema importância abordar a temática sobre obesidade na infância, pois essa doença está relacionada a diversas comorbidades, sendo desta forma, considerada um problema de Saúde Pública na atualidade, pois vem aumentando 
gradualmente os índices de morbidade e de mortalidade de crianças em idade préescolar - entre 4 a 6 anos - gerando doenças crônicas, como, por exemplo, doenças cardiovasculares, intolerância à glicose, dislipidemia, além da abnegação na vida psicossocial da criança, como: depressão, insatisfação com o próprio corpo, frustração em relação ao vestuário, discriminação e isolamento, dificuldade na higiene corporal, problemas respiratórios (hipoventilação ou dispneia) e apneia do sono (GASPARI, HERMAN, 2010).

Vale ressaltar que a obesidade infantil pode estar relacionada a diversos fatores, os quais se podem citar o sedentarismo, desmame precoce, maus hábitos e distúrbios alimentares. Existem casos de hipertensão arterial, dislipidemias e doenças cardiovasculares na infância, como complicações da obesidade (GODOI, 2013).

Além disso, A Organização Pan-Americana de Saúde (OPAS) e Organização Mundial de Saúde (OMS) afirmam que a obesidade infantil apresenta dimensões epidêmicas. No mundo, existem 17,6 milhões de crianças obesas com idade menor que cinco anos. O número de crianças obesas entre 6 a 11 anos desde a década de 60 dobrou. Trata-se de um problema global que atinge os países desenvolvidos de forma crescente, sendo responsável por 2 a $6 \%$ do custo total de atenção à saúde. Há certa valorização da corpulência como sinônimo de bebê saudável e bem cuidado em quase todos os grupos sociais, o que evidencia que a população desconhece ou banaliza a ocorrência da obesidade em bebês. Somado a isso estão às evidências de que crianças obesas tendem a se tornarem adultos obesos (ARAUJO et. al., 2006).

Contudo, estudo acerca da relação entre amamentação e obesidade infantil verificou que crianças que tiveram introdução precoce de alimentos complementares tinham significativamente uma média de Índice de Massa Corpórea maior do que as que receberam amamentação exclusiva (VAFA et. al., 2012).

Faz necessária a participação, nesse contexto, da enfermagem como sendo a profissão da área de saúde, ligada primeiramente a população com o objetivo da prevenção, e as demais com o tratamento, com isso torna-se indispensável à divulgação das atividades desenvolvidas pelo profissional de enfermagem, e as 
ações educativas, quanto à obesidade infantil, com intuito de prevenir que essas crianças se tornem obesa (TASSIANO, 2008).

Diante dos elevados índices de obesidade e das consequências que ela provoca os profissionais da saúde/enfermagem devem realizar seu diagnóstico precoce e programar ações de prevenção e controle desta enfermidade. No caso das crianças a resolução desse problema deve incluir informações e o aconselhamento da família sobre hábitos alimentares e prática de atividade física diária. Isso pode ser feito em diferentes contextos como escolas, comunidades ou Unidades de saúde (ARAÚJO et. al., 2012). Nesse sentido, este estudo permitiu uma visualização em destaque para a importância da família junto ao processo de prevenção, acompanhamento e fortalecimento das estratégias de tratamento da criança com obesidade. Isso porque a maior parte dos estudos descreve como atuação do enfermeiro no cuidado a criança obesa ações pactuadas com as famílias.

Evidências científicas revelam que diversas doenças típicas do adulto, começam a se desenvolver na infância, devido aos erros nos hábitos de vida, que contribuem para problemas futuros. Sendo assim, hábitos de vida saudáveis iniciados ainda na infância, são importantes estratégias para a prevenção de doenças crônico-degenerativas na vida adulta (BRASIL, 2014). Os estudos dessa pesquisa permitiram perceber que a atuação do enfermeiro na assistência e na educação em saúde permite auxiliar na disciplina alimentar por parte da família, sendo fundamental que a educação alimentar esteja presente desde a infância, à implantação de bons hábitos alimentares poderá evitar complicações futuras.

Em razão disto, os cuidados de prevenção e tratamento da obesidade é fundamental na atenção primaria. As intervenções de promoção à saúde, como orientar os pais sobre as mudanças da alimentação saudável e o incentivo à atividade física, desenvolvida pelos profissionais da saúde, tem repercutido diretamente na diminuição de morbimortalidade (FERNANDES; CONTERATO; MELLO, 2012). Nesse sentido, destaca-se a atuação do enfermeiro nesse nível de atenção a saúde e a evidência que os estudos analisados nessa pesquisa permitem compreender a atuação deste profissional como fortemente influente na equipe de 
saúde da Atenção Básica e capaz de realizar os cuidados a essa população específica.

Dessa forma é de suma importância que as estratégias de conscientização da população incluam também as escolas, através do uso de palestras educativas. Entretanto, outras estratégias podem ser realizadas para aperfeiçoar o processo de absorção de informações como dinâmicas interativas com alunos e professores com temas relacionados à alimentação saudável e prática de atividade física, capacitação das cozinheiras sobre os alimentos mais recomendados para prevenção da obesidade, exploração de meios artísticos como músicas, filmes e teatros educativos (ARAÚJO et al., 2006).

Ademais, o Ministério da Saúde destaca que as crianças ganham influências do meio em que convivem. A cultura dos hábitos alimentares é impactante na culpabilização da obesidade. Compete ao profissional de enfermagem, dentro do domínio de sua atuação, destacar como de fundamental importância o estabelecimento de propostas no sentido de sempre orientar acerca dos riscos que a doença promove (DAMASCENO, 2009).

No tocante ao aumento do número de obesos juvenis, os cuidados com essa população vêm se tornando desafiadores em todas as fases da vida, bem como a dificuldade de manter a disciplina alimentar. Concomitantemente, a multidisciplinaridade dos membros da equipe de saúde é de grande importância para conquistas futuras. Também é evidente que essas medidas só serão alcançadas se houver participação da família, das escolas e das comunidades, em um esforço conjunto da sociedade e do governo (LUNA IT et al., 2011). Tendo a enfermagem o papel instruir acerca das dietas alimentares e da prática de atividades físicas, encorajando e apoiando os pacientes por meio de ações educativas, durante todo o período de prática das ações de prevenção e promoção dos riscos a obesidade (KRUSE L, HENRIQUETA M et al., 2012).

Outro aspecto observado na analises dos estudos dessa pesquisa foi à necessidade de capacitação para os enfermeiros atuarem na detecção precoce, no acompanhamento, tratamento e reabilitação das crianças com obesidade, pois para o Ministério da Saúde a falta de capacitação da equipe pode ser um dificultador no acolhimento às pessoas com obesidade, uma vez que é imprescindível que os 
profissionais da Atenção Primária à Saúde (APS) estejam aptos e sensibilizados para captação e acolhimento eficientes dessa demanda.

Contudo, diante dos estudos analisados pode-se perceber que há necessidade de consultas de enfermagem cuidadosa no sentido de acompanhamento do crescimento e desenvolvimento das crianças para evitar a maior incidência de adolescentes e adultos obesos e o aumento das doenças crônicas não transmissíveis, priorizado sempre os métodos de prevenção das doenças e promoção da saúde.

\section{CONSIDERAÇÕES FINAIS}

Diante do exposto, pode-se dizer que, para a diminuição da obesidade que atualmente acomete crianças de todas as faixas etárias, é necessário frisar a enfermagem como parte contribuinte para diminuição desse agravo.

A enfermagem demonstrou ter um papel importante no cuidado à criança obesa e que o enfermeiro como educador em saúde e participante de uma equipe multiprofissional de saúde deve intervir e possibilitar uma melhor qualidade de vida a população, buscando apoiar a família, a escola e a comunidade para que todos possam participar ativamente da diminuição da obesidade infantil, prevenindo-os dos riscos e impedindo essas crianças de se tornarem um adulto obeso, e consequentemente vítima de outros agravos.

A enfermagem foi descrita como profissão capaz de atuar nesse sentido uma vez que exerce o cuidado direto da criança nas consultas de enfermagem, nas ações preventivas de educação em saúde nas escolas e na comunidade em geral, bem como no acompanhamento e tratamento de casos já detectados a fim de reverter um quadro de obesidade infantil e prevenir o aparecimento de doenças crônicas não transmissíveis. Contudo, foi possível perceber também a necessidade de capacitação para esses profissionais de saúde atuar com propriedade nessa área específica de saúde. 
O enfermeiro deve contribuir no cuidado da obesidade infantil, bem como, detectar os fatores de risco, atuando sempre na prevenção e promoção da saúde das crianças obesas, buscando conter o aumento exponencial da obesidade infantil no Brasil e no mundo e evitar o desenvolvimento das doenças associadas à obesidade.

\section{REFERÊNCIAS BIBLIOGRÁFICAS}

ALVES N.S.S.; FAUSTINO, T.K.A. Assistência de Enfermagem na Obesidade Infantil: Uma Revisão Integrativa. Brasília, 2019.

ARAUJO et al., Obesidade infantil: uma reflexão sobre dinâmica familiar numa visão etnográfica. Ceará: Universidade Federal do Ceará, 2006.

ARAÚJO S.N.M. et al. Obesidade infantil: conhecimentos e práticas de enfermeiros da Atenção Básica. Enfermagem em Foco, Piauí, v.3, n. 3, p. 139-142, 2012.

BRAGA V.A.S. et al. Intervenções do enfermeiro às pessoas com obesidade na Atenção Primária à Saúde: revisão integrativa. Revista da Escola de Enfermagem da USP. São Paulo, v. 51, mar. $2017 . \quad$ Disponível em: <http://www.scielo.br/pdf/reeusp/v51/pt_1980220XreeuspS1980220X201701920329 pdf>. Acesso em: 15 de fevereiro de 2020.

BRASIL. Ministério da Saúde; Secretaria de Atenção à Saúde, Departamento de Atenção Básica. Estratégias para o cuidado da pessoa com doença crônica: obesidade. Brasília: MS; 2014. (Cadernos de Atenção Básica, n. 38).

CARVALHO E.A.A. et al. Obesidade: Aspectos epidemiológicos e prevenção. Rev. Med. Minas Gerais, v. 23, n. 1, p. 74-82, 2013.

CORGOZINHO, JNC; RIBEIRO, G C. Registros de enfermagem e o enfoque na prevenção da obesidade infantil. RECOM, São João Del Rei- MG, v. 03, n. 03, p. 863- 872, 2013.

DAMASCENO, L.A. O papel do Enfermeiro na Prevenção da Obesidade Infantil: uma revisão bibliográfica. Disponível em: <http://luzidamasceno.blogspot.com.br/2009/12/opapel-doenfermeiro-na prevencaoda_17.html.>. Acesso em: 15 de fevereiro de 2020.

FERNANDES S.P.; CONTERATO E.V.; MELLO E.D. Manejo do Paciente Obeso Pediátrico na Atenção Primaria: Proposta de uma abordagem terapêutica prática. Rev. HCPA, v. 32, n. 4, 2012.

FREITAS A.S.S.; COELHO S.C.; RIBEIRO R.L. Obesidade infantil: influência de hábitos alimentares inadequados. Saúde\&Ambiente. Revista Duque de Caxias, v.4, n.2, p.9-14, 2009.

FULTON J.E. et al. Television viewing, computer use, and BMI among U.S. children and adolescents. J PhysAct Health, v. 6, n. 1, p.28-35, 2009.

GASPARI P.J.; HERMAN S. Atenção à saúde da criança: prevenção da obesidade infantil. Trabalho de Conclusão de Curso apresentado ao Curso de Enfermagem do Centro de Ciências da Saúde da Universidade Regional de Blumenau. Centro de Ciências da Saúde. Departamento de Enfermagem. 2010. 
KRUSE L. et al. Saúde e obesidade: discursos de enfermeiras. Aquichán, Bogotá, Aug, v. 12, n.2, 2012.

LINHARES F.M.M. et al. Obesidade infantil: influência dos pais sobre a alimentação e estilo de vida dos filhos. Temas em Saúde: João Pessoa, v. 16, n. 2, p. 460-481, 2016.

LUGÃO, M.A.S. et al. A importância da atuação do enfermeiro na prevenção da obesidade infantil. Rev. Cuidado Fundamental Online, v. 2, n. 3, p. 976-988, 2010.

LUNA I.T. et. al. Obesidade juvenil com enfoque na promoção da saúde revisão integrativa. Revista Gaúcha Enfermagem. (Online), v. 32, n2, p.394-401,2011.

MINISTÉRIO DO PLANEJAMENTO, ORÇAMENTO E GESTÃO. Pesquisa de orçamentos familiares 2008-2009: análise do consumo alimentar pessoal no Brasil / IBGE, Coordenação de Trabalho e Rendimento. - Rio de Janeiro: IBGE, p. 150, 2011.

MARCHI-ALVES, L. M. et al. Obesidade infantil ontem e hoje: importância da avaliação antropométrica pelo enfermeiro. Escola Anna Nery, São Paulo, v. 15, n. 2, p.238-244, 2011.

MARTINS T.A. et al. Fatores de riscos metabólicos em crianças na atenção primária à saúde. Rev baiana enferm. v.32, 2018.

ORGANIZAÇÃO PAN-AMERICANA DA SAÚDE. Doenças crônico-degenerativas e obesidade: estratégia Mundial sobre alimentação saudável, atividade física e saúde. Brasília, DF, 2003.

OLIVEIRA G.S. et al. Obesidade Infantil: Contribuição da Enfermagem na Prevenção. Revista Humano Ser - UNIFACEX, Natal, v.1, n.1, p. 1-12, 2016.

PINHO G.B.P.; GODINHO J.S.L. Assistência de enfermagem na prevenção e promoção da Saúde da criança e adolescente com risco para obesidade. Revista Pró- UniverSUS, v.8, n.2, p.10-14, 2017.

REY-LÓPEZ J.P. et al. Sedentary behaviour and obesity development in children and adolescents. Nutr Metab Cardiovasc Dis, v.18, p.242-251, 2008.

RIBEIRO K.R.A. et al. Ações da enfermagem no combate à obesidade infantil no período escolar. Revista Recien. São Paulo, v.5, n.15, p.11-18, 2015.

SANTOS, F.D.R. et. al. Ações de enfermeiros e professores na prevenção e no combate a obesidade infantil. Rev. Rene, v. 15, n. 3, p. 463-470, 2014.

SANTOS V.E.A. Prática de hábitos saudáveis na escola: atividade física, alimentação e controle de peso corporal infantil. Ariquemes: UnB; 2013.

SOUZA S.F.; SOUZA L.N. Orientações de enfermagem sobre prevenção da obesidade infantil. São Paulo: Revista Recien, v.5, n. 13, p.44-49, 2015.

SILVA, A.A.A. Saúde do escolar: Enfermagem na prevenção da obesidade infantil no contexto escolar. Gama-DF, 2019.

SILVA Y.M.P.; COSTA R.G.; RIBEIRO R.L. Obesidade Infantil: uma revisão bibliográfica. Saúde e Ambiente em Revista, v.3, n. 1, p.01-15, 2008.

VAFA M. et al. Relationship between breastfeeding and obesity in childhood. J Health Popul Nutr, 2012. 